\title{
Expectancies as mediators in the differential-reward conditional discrimination performance of pigeons
}

\author{
GAIL B. PETERSON \\ University of Minnesota, Minneapolis, Minnesota 55455 \\ RUTH L. WHEELER \\ Grinnell College, Grinnell, Iowa 50112 \\ and \\ GORDON D. ARMSTRONG \\ University of Minnesota, Minneapolis, Minnesota 55455
}

\begin{abstract}
In a series of within-subject experiments employing a two-choice delayed conditional discrimination task, pigeons chose correctly more often when kind of correct choice and kind of reinforcer were perfectly correlated than when uncorrelated. Correct choice behavior fell to chance levels when the correlation was reversed or when it was removed by using only one kind of reinforcer. Implications for mediation theories are discussed, with the conclusion being that, although the possibility that instrumental mediators are present in this situation cannot be dismissed, the overall pattern of results indicates that classical mediators are of principal importance in this type of task.
\end{abstract}

In one variety of the conditional discrimination procedure, the animal chooses between two stimuli presented simultaneously on each trial. The "correct" stimulus of the pair varies from trial to trial depending upon which of two "conditional" cues was presented several seconds earlier. Because each conditional cue occurs on a random half of the trials, the animal can master this task only if the conditional cue activates processes that persist from offset of the conditional cue until presentation of the choice stimuli.

Brodigan and Peterson (1976) have provided some insights into the nature of the mediating processes which allow the delay interval to be bridged in this type of conditional discrimination. They trained a group of pigeons for which a correct choice following one conditional cue was reinforced with food and a correct choice following the other cue was reinforced with water. Performance of this consistent-reward group was superior to that of an inconsistent-reward group trained on the same procedure, but for which correct choices following both conditional cues were reinforced equally often with food and water. It was also noted that pigeons in the consistent-reward group responded to the con-

This research was supported in part by Grant MH29244 from the National Institute of Mental Health and by a grant from the Graduate School of the University of Minnesota. Reprint requests should be sent to: Gail B. Peterson, Department of Psychology, Elliot Hall, 75 East River Road, University of Minnesota, Minneapolis, Minnesota 55455. ditional cue with a response topography appropriate to the reinforcer that would be available for a correct response on the subsequent choice trial. Sharp, open-beak pecks occurred to the cue associated with food; slower, more sustained key contacts accompanied by swallowing movements occurred to the cue associated with water reward (cf. Jenkins \& Moore, 1973; Moore, 1973; \& Wolin, 1968).

The Brodigan and Peterson result is compatible with the theory that animals learn to expect specific rewards and that reward expectancies can serve as mediators (Trapold, 1970). According to this view, the pigeons which received consistentreward training learned a specific reward expectancy in connection with each of the conditional cues. When a given conditional cue was presented, its associated reward expectancy was activated and persisted throughout the delay interval so that distinctive stimulus-feedback from the expectancy could cue the correct response to the choice stimuli. The reinforcer-specific response topographies observed in this group may be one indicator of these expectancies. Inconsistent-reward training does not permit differential reward expectancies to develop to each conditional cue. Consequently, the pigeons trained in this way lack the added discriminative stimulation from reward expectancies and, therefore, perform at a lower level of proficiency.

Although this particular experimental design is a fairly recent development, the theoretical analysis 
has deep historical roots. In a discussion of anticipatory defense reactions, Hull (1930) drew attention to the potential cue properties of conditioned responses with his concept of "pure stimulus acts," i.e., "acts whose sole function is to serve as stimuli for other acts" (p. 515), and shortly thereafter applied the concept to the analysis of maze learning in rats (Hull, 1931). The idea was elaborated upon further by Osgood (1953) under the rubric of "representational mediation processes," and has become quite well known in the form of the $\mathrm{r}_{\mathrm{g}}-\mathrm{s}_{\mathrm{g}}$ mechanism and related constructs (e.g., Amsel, 1962; Hull, 1952; Spence, 1956). Generally speaking, this explanatory model sees classical conditioning as the fundamental process underlying the mediating response mechanism, in that the responses which become conditioned to the discriminative stimuli and later function as the mediators are fractional components of the responses elicited by the reinforcer. Thus, this classical mediating response hypothesis stresses the importance of the particular CS-US or stimulus-reinforcer relations in the learning situation.

The experiments reported here further explored the consistent-inconsistent reward effect. As noted above, the classical mediating response analysis stressed the importance of the stimulus-reinforcer relations. If this analysis is valid, within-subject manipulation of these relations would be expected to have profound effects upon conditional choice performance.

\section{EXPERIMENT 1}

In Experiment 1, the consistent- and inconsistentreward procedures were compared using a withinsubject design. Two pigeons were first trained on the inconsistent-reward procedure and were then switched to consistent reward. A third pigeon received training with consistent-reward, inconsistentreward, and consistent-reward procedures, in that order. According to the classical mediation analysis, the pigeon's performance on the choice trials should be best when they receive consistent-reward training.

\section{Method}

Subjects. The subjects were three racing homer pigeons obtained from a local pigeon breeder. They were housed in individual cages in a continuously illuminated, temperaturecontrolled pigeon colony room. The birds were maintained at $80 \%$ of their free-feeding weights. Water rations were given after each session and were adjusted as necessary to insure that each subject would take both food and water during the next day's session. Water rations varied between 5 and $20 \mathrm{ml}$ per day.

Apparatus. The main apparatus was a Grason-Stadler Model 1122 operant conditioning chamber. It was equipped with three translucent response keys, a food hopper, a water cup, and a continuously illuminated houselight. One-plane readout projectors (Industrial Electronic Engineers, Inc.) were mounted behind the response keys and made possible the presentation of color and line pattern stimuli. The water cup was made of $1.27-\mathrm{cm}$-diam brass rod. Controlled amounts of water were delivered through a cylindrical passage in the core of the brass rod to a small, cone-shaped cup drilled in its top surface. The water cup protruded $2.54 \mathrm{~cm}$ into the chamber from the center of a $4.45 \times$ $8.90 \mathrm{~cm}$ white translucent Plexiglas plate that was attached to the inside surface of the front wall in the lower right corner. When water was delivered to the cup, the Plexiglas plate was illuminated from behind with white light for $3 \mathrm{sec}$. The syringe driver which pumped the water (Davis Scientific Instruments, Model 130) was located outside the sound-attenuation chamber that encased the pigeon box. The food hopper contained mixed grain, and the syringe contained plain tap water. Experimental events were controlled by conventional electromechanical programming equipment located in an adjacent room. Each pigeon was observed daily throughout the experiment via closed-circuit TV to determine whether it pecked the key, ate food, and drank water on a regular basis.

Procedure. All birds were trained to eat from the food hopper, drink from the water cup, and peck a white center key for food or water reinforcement. During the preliminary keypeck training phase, the center key was illuminated with white light every $30 \mathrm{sec}$. In the absence of a keypeck, the white key light went off after $6 \mathrm{sec}$ and was immediately followed, with equal likelihood, by either a $3-\mathrm{sec}$ access to grain or $.2 \mathrm{ml}$ of water. If the pigeon pecked the key before the $6 \mathrm{sec}$ had elapsed, the key light went out and the reinforcer was presented immediately. Keypecks during the intertrial interval (ITI) had no effect. Each session of preliminary training consisted of 80 such trials.

When all subjects were responding reliably to the onset of the center key, training on the conditional simultaneous discrimination task was begun. A trial began with the presentaton of either a green or red light on the center key. Three pecks on the center key resulted in the offset of the color cue and the onset of the choice stimuli, vertical and horizontal lines, located on the two side keys. Trials terminated with a single peck to one of the choice stimuli. Vertical was "correct" on green trials, horizontal on red trials. (This task is also known as "symbolic" or "conditional" matching.) Correct choices resulted in either a 3-sec access to grain or $.2 \mathrm{ml}$ of water and advanced the program to the ITI. An incorrect choice produced no reward, but advanced the program to the 5-sec ITI, during which time all keys were dark and after which the next trial in the sequence began. Responses during the ITI reset the ITI clock, thereby delaying onset of the next trial by $5 \mathrm{sec}$.

Each session contained 80 trials. Order of trials was random with the following restrictions: (1) Each successive block of 16 trials contained 8 green and 8 red trials; (2) within each of these 8-trial subsets, the correct choice stimulus occurred equally often on the left and right side keys, i.e., four times in each position. There was a total of five such random orders, which were used interchangeably across sections.

During the delay conditions, the delay occurred between the offset of the conditional cue and the onset of the choice stimuli. All keys were dark during the delay period; responses during the delay had no effect.

Figure 1 illustrates the task and the consistent- and inconsistentreward procedures. Two subjects (B3 and B5) started training with inconsistent reward. In this procedure, a correct choice resulted in food on a random half of each kind of trial and in water on the other half. The third subject (G3) received initial training with consistent reward where the kind of reward was perfectly correlated with the kind of trial, e.g, green-vertical-food and red-horizontal-water.

B3 and B5 were trained under inconsistent reward at 0-sec delay (zero delay) for 30 sessions, followed by 15 sessions at a 3-sec delay, and then a return to the zero delay condition for another 15 sessions. Following this, both animals were switched to con- 

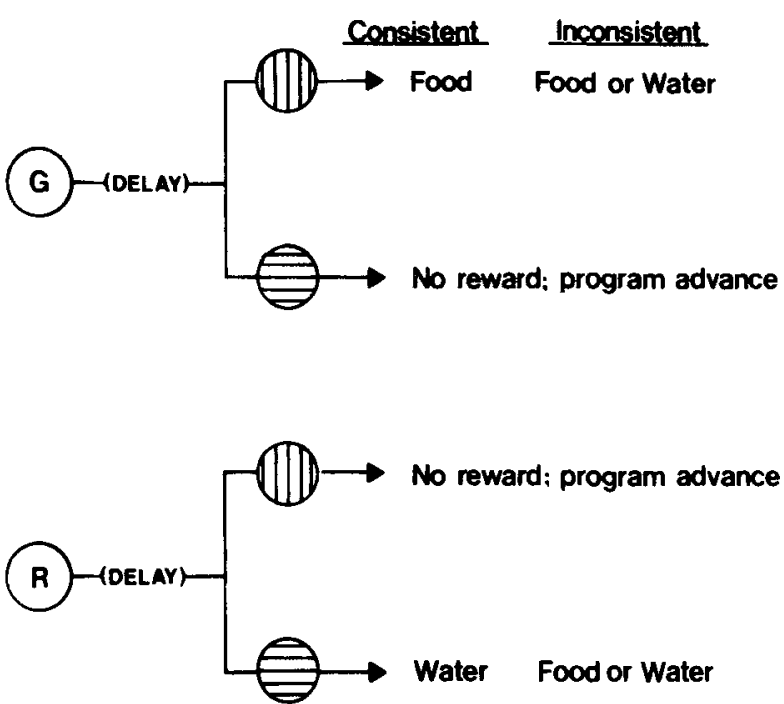

Figure 1. Schematic diagram of the consistent-reward and the inconsistent-reward training procedures.

sistent reward at zero delay. Subject B5 received 10 sessions of training under these conditions, after which the delay was extended to $2 \mathrm{sec}$ for 20 sessions, followed by 15 sessions of training with a 3 -sec delay. Subject B3 received 25 sessions of training at zero delay under consistent reward before the delay was extended to $2 \mathrm{sec}$ for 15 sessions after which training was discontinued for this subject.

Subject G3 received extensive training (over 125 sessions) with consistent reward in connection with another experiment (Armstrong, 1977), the methods of which did not disqualify it for the present work. Following training at zero delay, G3 was trained at a 3-sec delay for 9 sessions. Then, still at the 3-sec delay value, G3 was switched to inconsistent reward for 12 sessions. This was followed by a return to consistent reward at the 3 -sec delay for 15 sessions, after which training was discontinued.

\section{Results}

The top half of Figure 2 shows that B5 learned the problem on the inconsistent-reward procedure at zero delay, whereas B3 did not. The performance of B5 represents a more typical finding under inconsistent-reward conditions; that is, there is some mastery of the conditional discrimination, although the asymptotic level reached may be less than that of consistent-reward birds.

The performance level of B5 deteriorated rapidly when placed on the 3-sec delay, suggesting that the mechanism that was operative under zero delay either did not transfer to or was ineffective in the longer delay sessions. When B5 was returned to zero delay, correct choice performance rapidly returned to the original asymptotic level. B3 never exceeded chancelevel performance while under the inconsistentreward condition. Chance performance for both subjects was the result of a position preference for the left key response.

When switched to consistent reward, the performance level if both birds increased relative to their terminal performance under inconsistent reward. The subsequent delay conditions resulted in an initial decline in performance, followed by rapid improvement. It should be noted that consistentreward delay performance exceeded inconsistentreward zero delay performance for both birds.

The data obtained from $\mathrm{G} 3$ are illustrated in the bottom half of Figure 2. As noted above, this subject had received over 125 sessions of training with consistent reward and, therefore, only the last two blocks of training at zero delay are presented in the leftmost panel of the figure. Extending the delay to $3 \mathrm{sec}$ initially disrupted performance, but this was followed by a steady recovery. Whatever mechanism was responsible for this subject's relatively high level of performance under consistent reward, it was substantially affected by shifting to the inconsistent-reward procedure. The deterioration in performance was due to the development of a position preference (right key). When the consistentreward condition was restored, the performance level rapidly recovered.

\section{EXPERIMENT 2}

In Experiment 1, the importance of a consistent relationship between the kind of trial and kind of reward in promoting high levels of discrimination
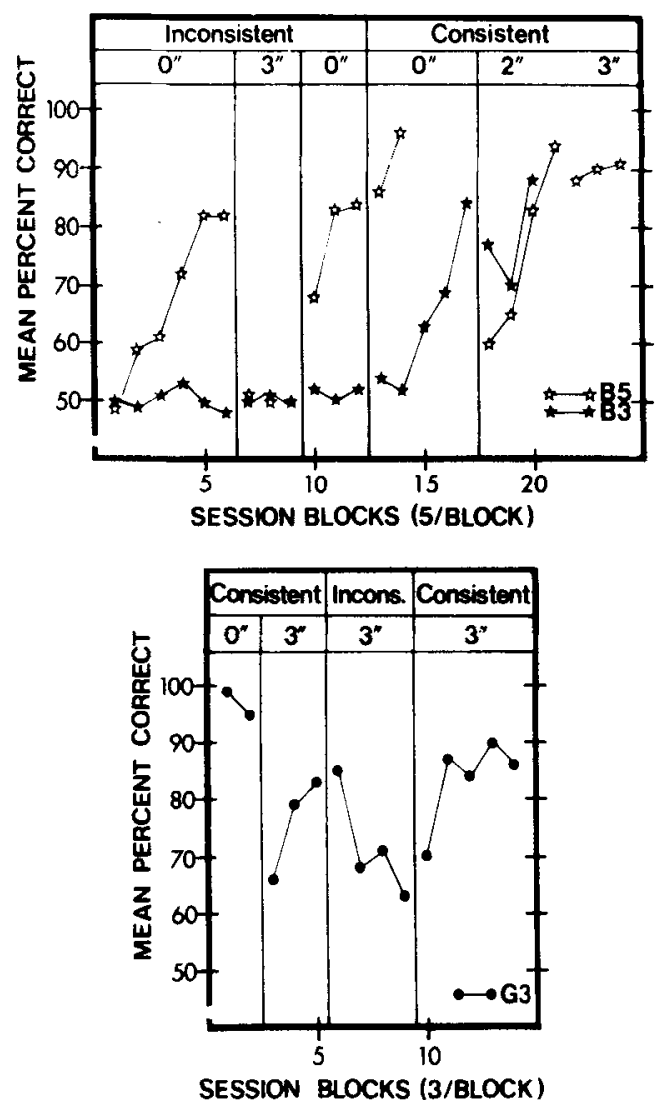

Figure 2. Mean percent correct choice responses over sessions for the three pigeons of Experiment 1 as a function of the training procedure and the delay between offset of the conditional cue and onset of the choice stimuli. 
performance was demonstrated by switching from the consistent- to the inconsistent-reward procedure, and vice versa. In Experiment 2, the problem was further explored by the reversal of the consistent outcomes. That is, following training to asymptote on consistent reward, the relation between the two conditional cues and food and water reward was simply reversed. The reversal manipulation retains the consistent-reward feature of the training procedure and does not confound the changing of specific cue-reward relations with the changing of the overall procedural format, a confounding which was present in Experiment 1.

\section{Method}

Subjects. The subjects were four experimentally naive racing homer pigeons. They were housed and maintained in exactly the same way as the subjects of Experiment 1.

Apparatus. The apparatus was that employed in Experiment 1.

Procedure. Preliminary training was the same as in Experiment 1 . When all subjects were responding well to the onset of the center key and eating and drinking reliably, they were transferred to the conditional discrimination task at zero delay under the consistent-reward procedure. All aspects of this task were the same as in Experiment 1. For two subjects, correct responses on green trials yielded food and correct responses on red trials yielded water, while for the other two subjects, water was the reward on green trials and food the reward on red trials.

Delay training was begun when a subject's performance equaled or exceeded $90 \%$ correct for three successive sessions. Upon reaching this criterion, two subjects (Bla and $\mathrm{B6}$ ) were placed on a 3-sec delay procedure and remained at that delay value for the rest of the experiment. The other two subjects (B7 and B8) received a more varied treatment at this point, being trained with delays which varied randomly across sessions among the values $0,1.5,3.0$, and $5.0 \mathrm{sec}$. The original purpose of this procedure was to determine if the temporal duration of the mediators in this situation was a significant factor. It proved not to be. Therefore, the delay was ultimately fixed at $3 \mathrm{sec}$ for these subjects also. All subjects were trained on the fixed 3-sec delay procedure for at least 12 sessions and until they obtained $90 \%$ or more correct on three successive sessions or until 45 sessions of training had been given under these conditions.

When the above criteria were met, the stimulus-reinforcer relations were reversed so that those stimulus-response sequences which had yielded food reward now yielded water, and vice versa. Note that the specification of the correct choice was not changed; choice of vertical lines following green and horizontal lines following red remained "correct." Training was continued on this procedure for 12 sessions unless average performance for a three-session block fell below chance ( $50 \%$ correct).

Following training on the outcome-reversal procedure, all subjects were retrained on their original consistent-reward procedure until they obtained $90 \%$ or more correct for three successive sessions or until 21 sessions of this recovery training had been administered.

\section{Results}

Figure 3 shows that the outcome-reversal procedure produced a return to chance-level performance in all four birds. However, complete deterioration of performance was not immediate. During the session immediately preceding outcome reversal, performance averaged $95 \%$ correct (range
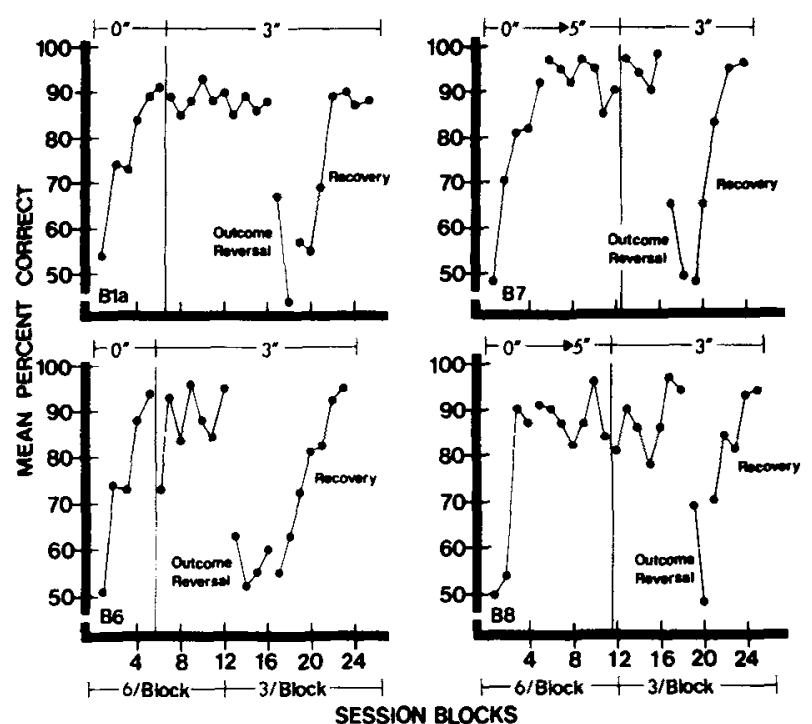

Figure 3. Mean percent correct choice responses over sessions for the four pigeons of Experiment 2 during consistent-reward training, outcome-reversal training, and recovery under consistent-reward training. The length of the delay interval is indicated at the top of each bird's graph. The data of principal interest are presented to the right of the vertical line in each panel. The data to the left of the vertical line are from initial acquisition with consistent reward under zero delay and, for $B 7$ and $B 8$, different delay values before the delay was set at 3 sec and the outcome-reversal manipulation was made. Breaks in the curves mark the points at which the indicated changes in conditions were made.

$89 \%-100 \%$ ). On the first session of outcome reversal, performance averaged $75 \%$ correct (range $65 \%-85 \%$ ). Although this clearly represents a decrement in performance, the absolute level is still well above chance. Despite this, correct choice behavior continued to decline. Indeed, its descent to chance level was quite rapid, with all birds performing at this level or very near it within six sessions. In all four birds, chance performance during outcome reversal was due to the development of a preference for the choice stimulus which (when correct) yielded food. The recovery functions shown for all birds represent the result of the return to their original training conditions.

\section{EXPERIMENT 3}

The results of Experiments 1 and 2 indicated that, once successful choice behavior across delays is established via consistent-reward training, switching to inconsistent reward or outcome-reversal procedures greatly disrupts efficient choice performance. These findings are consistent with the rewardexpectancy mediation analysis outlined above. The conventional training procedure in conditional discrimination studies utilizes only a single type of reinforcer, typically food, which is given following correct choices on both kinds of trials. Experiment 3 
determined the effect of switching to this conventional, single-reinforcer procedure following consistentreward training. In addition, one subject received outcome-reversal training as a further check on the reliability of the Experiment 2 finding.

\section{Method}

Subjects. The subjects were one racing homer obtained from a local breeder and one wild pigeon captured locally. They were housed and maintained under the same conditions as the subjects of Experiments 1 and 2. The racing homer (subject G1) had participated in another experiment (Armstrong, 1977) and had received extended training under the consistent-reward procedure at delay values up to $10 \mathrm{sec}$. The wild pigeon (subject GDA1) also had an extensive history of consistent-reward training, having served as a subject in Brodigan and Peterson's (1976) study and having received additional training at delay values up to $10 \mathrm{sec}$. None of this previous experience, however, disqualified the subjects for the purposes they served in the present study.

Apparatus. The apparatus was that used in Experiments 1 and 2.

Procedure. Before the single reinforcer manipulations of the present experiment were made, each subject's performance had been stable for several sessions at $90 \%$ to $100 \%$ correct on the color/line tilt conditional discrimination task under the consistentreward procedure at a 10 -sec delay. All of the manipulations in this experiment were made at the 10 -sec delay value. Other aspects of the procedure were the same as in the previous experiments.

Subject G1 was first changed to a procedure on which water was given for correct responses on both red and green trials, food reward being discontinued entirely. After six sessions on this procedure, $\mathrm{Gl}$ was returned to its original consistent-reward procedure. When the criterion of three successive sessions at $90 \%$ or more correct had been recovered, G1 was placed on a procedure which yielded only food reinforcers for correct choice responses on both kinds of trials. Following nine sessions on the food-only procedure, the consistent-reward procedure was reinstated until criterion performance was recovered. Finally, G1 was exposed to the outcome-reversal procedure of Experiment 2 for six sessions, after which its performance on the original consistent-reward procedure was recovered once again.

Subject GDAl was first tested under the food-only procedure for 6 days. During this test and during the water-only and foodonly testing of $\mathrm{G} 1$, the subjects had been on their normal food and water deprivation scinedules. In order to determine if the effect of the single-reinforcer manipulation was related to the combined deprivation conditions, training was continued with GDA1 for 12 additional sessions with food as the reward but, in addition, water was freely available in the home cage. This test was followed by a return to food and water deprivation and the consistent-reward procedure until the $90 \%$ criterion had been recovered, after which the experiment was terminated.

\section{Results}

Figure 4 presents the data from the two birds, beginning with their terminal performance at a 10-sec delay on the consistent-reward procedure followed by the outcome of the manipulations made at the 10 -sec delay level.

Subject G1's data, shown in the left panel of Figure 4, replicate the outcome-reversal findings reported above with a 3 -sec delay and, in addition, demonstrate the effect of changing to one type of reward for all correct choices (i.e., water only or food only). The outcome reversal manipulation pro-

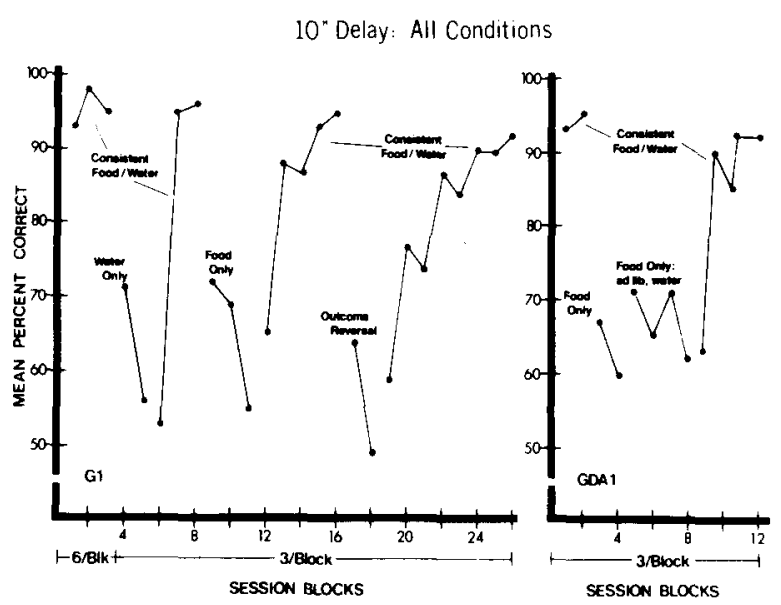

Figure 4. Mean percent correct choice responses over sessions for the two pigeons of Experiment 3 during consistent-reward training and single-reward training. Subject G1 was also tested under the outcome-reversal condition. The delay between conditional cue offset and choice stimuli onset was 10 sec during all phases of the experiment.

duced the greatest disruption of correct choice behavior, both in terms of the performance level exhibited during the manipulation and the number of sessions required for recovery. The reduced levels of choice performance following the various procedural changes were the result of different patterns of behavior. Under the water-only condition, G1 developed a position preference for the right response key. Under the food-only condition, correct and incorrect choice responses were uniformly distributed across response keys and choice stimuli. As had been observed in Experiment 2, chance performance under the outcome-reversal condition was the result of the subject's persistent choice of the stimulus associated with food.

The data of subject GDAl are presented in the right panel of the figure and conform to the same pattern as those of G1. Performance improved somewhat from the initial decline to chance level when GDA1 was taken off water deprivation, but the behavior was quite variable and substantially below that demonstrated in the subsequent recovery phase. Low levels of performance by this subject were primarily due to a position preference, although on some sessions correct and incorrect choices were uniformly distributed across response keys and choice stimuli.

\section{DISCUSSION}

Following consistent-reward training, inconsistentreward, outcome-reversal, and single-reward training produced substantial decrements in correct choice behavior, almost always to chance levels. This pattern of results is consistent with the classical 
mediating response analysis outlined in the introduction. That analysis stresses the stimulusreinforcer relation as a source of the mediating process that bridges the temporal gap between offset of the conditional cue and presentation of the choice stimuli. Thus, when the consistent cue-reward relation was changed in the inconsistent-reward, outcome-reversal, and single-reward procedures, the mediating mechanism broke down and correct choice performance declined.

The results of these experiments also speak to another version of mediating response theory which, as noted by Brodigan and Peterson (1976), might be applied to the consistent-inconsistent reward effect. This version may be referred to as the instrumental mediating response hypothesis and can be thought of as an interpretation of Hull's concept of pure stimulus acts in which the "acts" are instrumental or operant responses rather than Pavlovian CRs. According to this hypothesis, the two conditional cues are the initial SDs for chains of instrumental responses which culminate in a response to the "correct" choice stimulus associated with that cue. These behavior chains are acquired and maintained via the law of effect because they are instrumental in obtaining reinforcers. Blough (1959) appears to have been the first to suggest this hypothesis in connection with delayed conditional discrimination performance in pigeons, but the same idea has been expressed by numerous other workers (e.g., Cumming \& Berryman, 1965; Cumming, Berryman, \& Cohen, 1965; Lawrence, 1963; Schoenfeld \& Cumming, 1963). In support of the hypothesis, recent evidence has indicated that differential response requirements (defined either topographically or by schedule) to the cues in conditional discrimination tasks promote higher levels of discrimination performance than are obtained under nondifferential procedures (e.g., Cohen, Looney, Brady, \& Aucella, 1976; Eckerman, 1970; Shimp \& Moffitt, 1977).

Application of this hypothesis to the consistentinconsistent reward effect would require the consistent-reward procedure to induce (cf. Segal, 1972) differential instrumental chains that are more discriminable from one another than those acquired under the inconsistent-reward procedure. As noted above, distinctive response topographies have been observed to the conditional cues in the present type of experiment when consistent-reward training is given, but not when training involves inconsistent reward (Brodigan \& Peterson, 1976). However, these responses seem related to the nature of the reinforcer signaled by the cue and appear to originate from stimulus-reinforcer associations rather than from instrumental response-reinforcer relations (cf. Jenkins \& Moore, 1973; Moore, 1973). Still, it is possible that there is an instrumental component to the mediating process in the present type of experiment. For example, behaviors other than the topographically distinct contact responses may be associated with the two conditional cues, and these may arise from the response-reinforcer relation. Also, the eating and drinking topographies may themselves be modified by their instrumental relation to the (delayed) reinforcer. Whatever the case, it would seem that an account of the present data solely in terms of instrumental mediating responses is inadequate. Once different instrumental chains had been established, merely changing the kind of positive reinforcer which terminated them on half of the trials (Experiments 1 and 3 ) or even on all of the trials (Experiment 2), while all other conditions (e.g., discriminative stimuli, response requirement, drive state, etc.) remained constant, should not have resulted in the chains' destruction, as the devastated choice performance suggests. The instrumental function of the mediators would not have changed, i.e., they would still lead to the correct response and it would lead to a reinforcer, so why would the mediating chains deteriorate? Thus, although the possibility that instrumental mediators may be present in this type of experiment cannot be dismissed entirely, if they are present the data suggest that they are of considerably less importance than classical mediators in terms of their control over correct-choice behavior.

Because of the response-requirement features of the procedure employed in the present experiments (i.e., three pecks were required to the conditional cue, one peck to the choice stimuli), this work is formally instrumental conditioning. In view of the data and foregoing discussion, however, it would be of interest to determine whether the keypecking and discriminative performance observed in these experiments were significantly dependent upon this procedural detail. It may be that the response requirements served merely to expose the subject to stimulus-stimulus contingencies which determined keypecking and discriminative performance, the response-reinforcer contingencies being totally unnecessary. The response-independent delayed conditional successive discrimination procedure developed recently by Wasserman (1976) might be adapted to explore this possibility. For instance, successive presentations of green-vertical and redhorizontal would be followed by food or water reinforcers (according to either the consistent- or inconsistent-reward format) while green-horizontal and red-vertical sequences would not be followed by reinforcers, all of this being done independently of the subject's behavior. Occasional test trials with the line patterns presented simultaneously following either red or green could also be given. Appropriate 
choice beavior by consistent-reward subjects but not by inconsistent-reward subjects would essentially replicate the findings reported here and indicate that the response-requirement features of the present experiment were unimportant. The results of such an experiment would also have implications for the role of response requirements in the conventional singlereward conditional simultaneous discrimination paradigm.

Another research problem pertains to the role played by the different overt behaviors evoked by the conditional cues in consistent-reward subjects. The presence of conspicuous US-specific responses, it should be noted, does not force the conclusion that the outcome expectancy must be peripheral. Different peripheral responses might be no more than epiphenomenal manifestations of different central responses. It would be interesting to know if the consistent-inconsistent reward effect obtains with discriminably different yet equally preferred reinforcers which elicit the same URs and condition identical CRs. Further, could discriminably different but putatively neutral stimuli be employed as outcome events to produce the effect? The answers to these questions would aid greatly in further specifying the nature of expectancies in animal learning.

\section{REFERENCES}

AMSEL, A. Frustrative nonreward in partial reinforcement and discrimination learning: Some recent history and a theoretical extension. Psychological Review, 1962, 69, 306-328.

Armstrong, G. D. Displacement behaviors of pigeons during a two-choice conditional discrimination problem. Unpublished doctoral dissertation, University of Minnesota, 1977.

Blough, D. S. Delayed matching in the pigeon. Journal of the Experimental Analysis of Behavior, 1959, 2, 151-160.

Brodigan, D. L., \& Peterson, G. B. Two-choice discrimination performance of pigeons as a function of reward expectancy, prechoice delay, and domesticity. Animal Learning \& Behavior, 1976, 4, 121-124.

Cohen, L. R., Looney, T. A., Brady, J. H., \& Aucella, A. F. Differential sample response schedules in the acquisition of conditional discriminations by pigeons. Journal of the Experimental Analysis of Behavior, 1976, 26, 301-314.
Cumming, W. W., \& Berryman, R. The complex discriminated operant: Studies of matching-to-sample and related problems. In D. I. Mostofsky (Ed.), Stimulus generalization. Stanford: Stanford University Press, 1965.

Cumming. W. W., Berryman, R., \& Cohen, L. R. Acquisition and transfer of zero-delay matching. Psychological Reports, $1965,17,435.445$.

ECKERMAN, D. A. Generalization and response mediation of a conditional discrimination. Journal of the Experimental A nalysis of Behavior. 1970, 13, 301-316.

Huls. C. L. Knowledge and purpose as habit mechanisms. Psychological Review. 1930, 37, 511-525.

Hull, C. L. Goal attraction and directing ideas conceived as habit phenomena. Psychological Review, 1931, 38, 487-506.

Hull. C. L. A behavior system. New Haven: Yale University Press. 1952

Jenkins, H. M., \& Moore, B. R. The form of the auto-shaped response with food or water reinforcers. Journal of the Experimental Analysis of Behavior, 1973, 20, 163-181.

LAWRENCE. D. H. The nature of a stimulus: Some relationships between learning and perception. In $S$. Koch (Ed.) Psychology: A study of a science (Vol. 5). New York McGraw-Hill, 1963.

Moore, B. R. The role of directed Pavlovian reactions in simple instrumental learning in the pigeon. In R. A. Hinde \& J. S. Hinde (Eds.), Constraints on learning. London: Academic Press, 1973.

OsGood, C. E. Method and theory in experimental psychology. New York: Oxford University Press, 1953.

Schoenfeld, W. N., \& Cumming, W. W. Behavior and perception. In S. Koch (Ed.), Psychology: A study of a science (Vol. 5). New York: McGraw-Hill, 1963.

SEGAL. E. F. Induction and the provenance of operants. In R. J. Gilbert \& J. R. Millenson (Eds.), Reinforcement: Behavioral analyses. New York: Academic Press, 1972.

Shimp, C. P., \& Moffitt, M. Short-term memory in the pigeon: Delayed-pair-comparison procedures and some results. Journal of the Experimental Analysis of Behavior, 1977. 28, 13-25.

SPENCE, K. W. Behavior theory and conditioning. New Haven: Yale University Press, 1956.

Trapold, M. A. Are expectancies based upon different positive reinforcing events discriminably different? Learning and Motivation, 1970, 1, 129-140.

WASSERMAN, E. A. Successive matching-to-sample in the pigeon: Variations on a theme by Konorski. Behavior Research Methods \& Instrumentation, 1976, 8, 278-282.

WoLIN, B. R. Differences in manner of pecking a key between pigeons reinforced with food and water. In A. C. Catania (Ed.), Contemporary research in operant behavior. Glenview, Ill: Scott, Foresman, 1968.

(Received for publication September 7, 1977; revision accepted February 16, 1978.) 